\title{
Knowledge Base Law: Ein neuer Weg bei der Wissensvermittlung juristischer Information
}

\author{
Michael Nentwich \\ Österreichische Akademie der Wissenschaften, Institut für Technikfolgen-Abschätzung \\ Strohgasse 45/5, 1030 Wien \\ mnent@oeaw.ac.at \\ Schlagworte: Datenbank, Open Content, elektronische Publikationen, Rechtsverglei- \\ chung, FAQ, Urheberrecht, Informationsgesellschaft \\ Abstract: Der Beitrag stellt das Konzept „Knowledge Base Law“ (KB:Law) vor. Es \\ handelt sich um ein innovatives Datenbanksystem, eine sog Wissensbasis. \\ Juristische Informationen werden sowohl für Laien als auch Experten zu- \\ gänglich gemacht, indem neben der Grundinformation stets auch eine umfas- \\ sende rechtliche Analyse zur Verfügung stehen wird. Die Antworten werden \\ in verschiedenen Sprachen und aus Sicht verschiedener Länder und deren \\ Rechtssystemen beantwortet sowie reichlich dokumentiert. KB:Law ist somit \\ eine multidimensionale FAQ-Datenbank, eine neuartige Publikationsmög- \\ lichkeit, aber auch ein Instrument der Rechtsvergleichung.
}

\section{Einleitung}

Die Knowledge Base Copyright Law" (kurz: KB:Law|C) wurde als ein Arbeitspaket im Rahmen eines Projekts, das vom Wiener Wissenschafts- und Technologiefonds (WWTF) gefördert wird, entwickelt. ${ }^{1}$ Anlässlich der IRIS'06 erfolgte der offizielle Launch der Applikation unter der Webadresse kb-law.info. Der folgende Beitrag stellt die implementierten Funktionalitäten im Überblick dar (2.1), verweist auf das Potenzial als juristisches Publikationsorgan (2.2) sowie als Instrument der Rechtsvergleichung (2.3) und erläutert die Zukunft des Projekts aus Sicht der Initiatoren (3).

\footnotetext{
${ }^{1}$ Zum Konzept des Projekts genauer in Trybus/Nentwich, 2005, Knowledge Base Copyright Law - Wissensbank Urheberrecht für alle? In: Schweighofer/Liebwald/ Menzel/Augeneder (Hg): Effizienz von e-Lösungen in Staat und Gesellschaft. Aktuelle Fragen der Rechtsinformatik. Tagungsband des 8. Internationalen Rechtsinformatik Symposions IRIS 2005, Boorberg, Stuttgart et al., 351-358.
} 


\section{Wesentliche Funktionalitäten}

\subsection{Mehrdimensionale FAQ mit Dokumentation}

Eine KB:Law ist sowohl eingabeseitig (Backend) als auch auf Seite der User (Frontend) eine datenbankbasierte Webapplikation für juristische Inhalte. Im Kern handelt es sich um eine Datenbank mit so genannten Frequently Asked Questions (FAQ). Auch in einer KB:Law werden Fragen beantwortet, die sich im jeweiligen Rechtsgebiet in der Praxis stellen. Der Zugang ist somit zunächst ein lebensweltlicher (bottom-up), kein systematischer (top-down). Im Unterschied zu herkömmlichen FAQ-Seiten werden die Antworten jedoch in einer ausgefeilten Hypertextstruktur präsentiert, die mehr bietet als bloß eine Antwort auf die Frage; eine KB:Law-Antwort auf eine bestimmte Frage stellt vielmehr ein Bündel an Antworten dar:

- Zunächst werden Antworten auf zwei Niveaus angeboten: Erstens in einer Überblicksfassung, die in einer für juristische "Laien“ verständlichen Sprache verfasst ist, die aber nichtsdestotrotz präzise Auskunft über die Rechtslage gibt; zweitens in einer detaillierten Langfassung, die sich primär an Juristen wendet, entsprechend dokumentiert ist und auch im Fachjargon geschrieben ist.

- Weiters werden die Antworten spezifisch für bestimmte Rechtsordnungen (Länder) gegeben. Dies hat den Vorteil, dass gerade bei nicht oder nur teilweise harmonisierten Rechtsbereichen auf die unterschiedlichen Rechtslagen eingegangen werden kann.

- Schließlich ist eine KB:Law mehrsprachig, dh die Antwort liegt in mehreren Sprachen vor.

Das Userinterface ermöglicht es, jederzeit mit einem Mausklick von einer Antwortfassung zur nächsten zu wechseln, also etwa von der Überblicksantwort zur Detailfassung, von der englischen Version zur deutschen oder von der Antwort für Österreich zu jener für die USA. $\mathrm{Zu}$ jeder Antwort gibt es weiters über spezielle Kontextbuttons zu erreichende Dokumentationen: Rechtsquellen (zB Gesetze, soweit vorhanden, im Volltext), Urteile und sonstige Entscheidungen (ebenso, soweit vorhanden, im Volltext) sowie Literaturzitate (soweit vorhanden auch mit Link zum Volltext). Zu den Fragen und Antworten kommt man über drei verschiedene Wege. Erstens über Fragenlisten: Im Backend, also während der Eingabe, werden alle Fragen sog Kategorien bzw Subkategorien (eventuell auch mehrfach) zugeordnet; nach diesen Zuordnungen werden die Fragen im Frontend gegliedert in Listenform dargestellt. Zweitens über eine Fragenvorauswahl nach Zielgruppen: Im Backend können verschiedenen Zielgruppen definiert und diesen 
dann Fragen zugeordnet werden; im Frontend gibt es dann zielgruppenspezifische Einstiegsseiten mit einem Einleitungstext und einer Auswahl von relevanten Fragen. Die dritte Möglichkeit ist ein Suchformular, das sowohl Volltext- als auch Stichwortsuchen sowie die Einschränkung nach Zielgruppen, Antwortebenen, Ländern und Sprachfassungen ermöglicht. Ein themenspezifisches Glossar ergänzt das Angebot.

\subsection{Neuartige Publikationsplattform}

Während KB:Law in erster Linie als ein Informationsinstrument für an praktischen juristischen Fragen Interessierte geplant war, kann man es aus Sicht der Verfasser der Antworten auch als neuartige Publikationsmöglichkeit ansehen. Vor allem die Detailfassungen der Antworten, die in der Regel 2-3 Druckseiten lang sind, können zweifellos als eigenständige Online-Veröffentlichung bezeichnet werden. Die Implementierung des Userinterfaces sorgt dafür, dass diese Antwortartikel immer unter einer eindeutigen Webadresse abrufbar bleiben und damit zitierfähig sind. Eine spezielle Druckfunktionalität bereitet die Artikel, die zunächst noch als Hypertext vorliegen (der Antworttext, die Fußnoten, die Literatur-, Rechtsquellen- und Entscheidungsregister werden in verschiedenen, miteinander verlinkten Bildschirmfenstern dargestellt), zum Ausdrucken in einer Datei auf, die auch in Form und Layout in etwa typischen juristischen Artikeln entspricht. Sie sind namentlich gekennzeichnet, tragen ein Versionsdatum und bieten am Kopfende einen Zitiervorschlag, der beispielsweise so aussieht:

Peter Trybus, Gibt es schöpferische Werke, die keinen urheberechtlichen Schutz genießen? Was sind "freie Werke"? (Detaillierte Antwort für Österreich),

Knowledge Base Copyright Law (KB:Law|C), Antwort Nr. 8, Version: 16.3.2006 14:01 http://kb-law.info/kbc/kbc.php?article=8\&land=AT\&lang=DE\&mode=1

Das Backend ermöglicht darüber hinaus ein rollenbasiertes Qualitätssicherungssystem: Autoren geben ihre Antworttexte online in das System ein, wobei die Texte nicht sofort frontendseitig abrufbar sind. Der Editor-in-Chief sorgt für eine entsprechende Qualitätskontrolle, etwa durch Zuweisung an einen Gutachter. Erst nach Einarbeitung eventueller Verbesserungsvorschläge und Korrekturen wird der Text „freigeschaltet“, also über das Frontend abrufbar gemacht.

\subsection{Instrument der Rechtsvergleichung}

Über die vorgenannten Zwecke eines Informationsinstruments (FAQ) und eines neuartigen Publikationsmediums hinaus kann 
KB:Law schließlich auch als Instrument der Rechtsvergleichung begriffen werden. Aufgrund der länderspezifischen Beantwortung spezieller Fragen wird der direkte und zielgerichtete Vergleich der Rechtslagen in zwei oder mehreren Ländern erleichtert. Die Möglichkeit der dezentralen Erstellung und Einspeisung von Antworten bietet ideale Voraussetzungen für länderübergreifende Kooperationsprojekte. Die Beantwortung sehr spezifischer (praktischer) Fragen (aus Anwendersicht) führt potenziell zur Aufdeckung konkreter Unterschiede in der Rechtslage bzw Anwendungspraxis und kann damit zum Ausgangspunkt für Harmonisierungsbemühungen werden.

\section{Status Quo und Ausblick}

Zum Zeitpunkt des Verfassens dieses Beitrags sind die verschiedenen Softwaremodule (Backend und Frontend) in Version 1.0 fertig gestellt, dh alle geplanten Funktionalitäten sind soweit implementiert, dass sowohl die Einspeisung von Inhalten im Backend als auch das Abrufen derselben im Frontend reibungslos funktioniert. Das Konzept KB:Law wurde aufgrund des Projektzusammenhangs (siehe unter 1) zunächst für den Bereich des Urheberrechts mit besonderem Schwerpunkt auf jenen Fragen, die sich hier in Hinblick auf die Informationsgesellschaft stellen, in einem Prototypen verwirklicht, in der Knowledge Base Copyright Law (KB:Law|C), siehe kb-law.info/kbc/kbc.php. Aktuell sind 52 Fragen in zumindest einer Sprachfassung (zumeist Deutsch, teilweise Englisch), auf einem Antwortniveau und für zumindest ein Land (zumeist Österreich, teilweise Deutschland und die USA) beantwortet sowie 50 Literaturstellen, 38 Urteile und 232 Gesetzesstellen erfasst. Es ist klar, dass $\mathrm{KB}: \mathrm{Law} \mid \subset \mathrm{C}$ bislang vor allem dazu dient, die Realisierbarkeit des Konzepts nachzuweisen, was aus unserer Sicht eindeutig gelungen ist.

In einem nächsten, nahe liegenden Schritt sollen die schon gestellten Fragen für möglichst viele weitere Länder beantwortet und übersetzt sowie durch weitere Fragen ergänzt werden, damit KB:Law|C auch als umfassende Wissensbasis seinen Zweck erfüllen kann. Das Projektteam ist bemüht sich auf zwei unterschiedlichen Pfaden, diesem Ziel näher zu kommen: Einerseits laufen Drittmittelanträge, die es uns ermöglichen würden, Juristen zu finanzieren, die systematisch Antworttexte einspeisen. Andererseits planen wir, über internationale Netzwerke und das Angebot einer innovativen Veröffentlichungsplattform potenzielle Autoren zu gewinnen. In der Zukunft wollen wir weitere KB:Laws in anderen Rechtsbereichen, etwa im Arbeits- oder Steuerrecht, initiieren. 